\title{
A fast and non-degenerate scheme for the evaluation of the 3D fundamental solution and its derivatives for fully anisotropic magneto-electro-elastic materials ${ }^{\text {th }}$
}

\author{
Federico C. Buroni ${ }^{\mathrm{a}, *}$, Cristiano Ubessi ${ }^{\mathrm{b}}$, Gabriel Hattori ${ }^{\mathrm{c}}$, \\ Rogério J. Marczak ${ }^{b}$, Andrés Sáez ${ }^{\mathrm{d}}$ \\ ${ }^{a}$ Department of Mechanical Engineering and Manufacturing, Universidad de Sevilla. \\ Camino de los Descubrimientos s/ $n$, Seville E-41092, Spain \\ ${ }^{b}$ Department of Mechanical Engineering - DEMEC/PROMEC, Universidade Federal do \\ Rio Grande do Sul. Sarmento Leite 425, 90050-160, Porto Alegre, Brazil \\ ${ }^{c}$ Department of Engineering, University of Cambridge, CB2 1PZ, Cambridge, UK \\ ${ }^{d}$ Department of Continuum Mechanics and Structural Analysis, Universidad de Sevilla. \\ Camino de los Descubrimientos s/n, Seville E-41092, Spain
}

\begin{abstract}
A new expression for the fundamental solution is introduced, presenting three relevant characteristics: $(i)$ it is explicit in terms of the Stroh's eigenvalues, (ii) it remains well-defined when some Stroh's eigenvalues are repeated, and ( $i i i)$ it is exact. A fast and robust numerical scheme for the evaluation of the fundamental solution and its derivatives developed from double Fourier series representations is presented. The Fourier series representation is possible due to the periodic nature of the solution. The attractiveness of this series solution is that the information of the material properties is contained only in the Fourier coefficients, while the information of the dependence of the evaluation point is contained in simple trigonometric functions. This implies that any order derivatives can be determined by spatial differentiation of the trigonometric functions. Moreover, Fourier coefficients need to be obtained only once for a given material, leading to an efficient methodology.
\end{abstract}

${ }^{\pi}$ Special issue on the Tribute of the Brazilian Computational Mechanics Community to Carlos Brebbia: Edited by Ney Augusto Dumont and José Telles

*corresponding author

Email address: fburoni@us.es (Federico C. Buroni) 
The robustness of the scheme arises from the properties $(i)$ and $(i i)$ of the new expression for the fundamental solution, which is used to compute the Fourier coefficients. The proposed approach combines the clean structure of the Stroh formalism with the simplicity of Fourier expansions, addressing the old drawbacks of anisotropic fundamental solutions.

Key words: explicit expressions, mathematical degenerate materials, Stroh formalism, three-dimensional magnetoelectroelasticity, Green's functions, Fourier series representation

\section{Introduction}

In the last few years, the Magneto-Electro-Elastic (MEE) coupling presented in composites consisting of Piezoelectric (PE) and Piezomagnetic (PM) phases has been the focus of intensive research due to the numerous emerging possibilities at multiple material scales. These coupling phenomena have also been used for modern applications in various devices: sensors, actuators and smart structures are just some of the current applications. New possibilities in fabrication and design of advanced materials of general anisotropy and novel micro/nano devices and structures require robust and efficient numerical methods for the analysis of anisotropic multicoupling materials. Historic developments, challenges and perspectives of MEE materials and its applications can be found in recent reviews $[1,2]$.

Fundamental solutions can be used to solve sophisticated boundary value problems. They are a central subject in modelling the physical and mechanical behaviour of solids. For instance, they are required to obtain stresses due to internal defects in materials. They are also required for the analysis of general problems by some numerical methods, such as the Boundary Element Method (BEM) [3-5], to which Professor Carlos Brebbia has made extensive contributions [6]. In all these methodologies, fundamental solutions are typically evaluated thousands or millions of times. Advances in the use of these numerical methods are limited by the availability of efficient and robust numerical schemes for the corresponding fundamental solutions. Additionally, these solutions require extensive computations, which can render the numerical method infeasible for large problems.

All multifield materials have some degree of anisotropy. Hence, the development of anisotropic fundamental solutions with coupled behaviour has been built upon previous research for purely elastic anisotropic elasticity his- 
torically. A recent comprehensive review of this development can be found in Muñoz-Reja et al. [7], so we focus our attention in the main drawbacks of existing three-dimensional (3D) anisotropic MEE solutions. The main interest in finding fundamental solutions in a suitable form for their numerical implementation has been the computational cost. For comparison purposes, the general anisotropic elastic case contains 21 different elastic constants, however the amount of calculations for the fundamental solution is already large. For the MEE case, with 75 different material constants, the computational cost becomes a key issue. In general, fundamental solutions are available in the literature either in integral form or in terms of explicit expressions.

On one hand, fundamental solution and its derivatives may be presented in integral form (see the work by Han [8] and references therein). This approach is called implicit and involves numerical integration of regular functions. When the integral part of the fundamental solution is arranged in order to be dependent only on the direction of the evaluation vector but not on its modulus (see for instance deduction by Wang \& Achenbach [9] for the elastic case), the smoothness of the kernels depends on the degree of anisotropy. In alternative integral forms, corresponding kernels may become less smooth and more difficult to integrate for small modulus of the position vector [10]. At any event, regarding the BEM implementation of the fundamental solutions, the numerical computation of the involved integrals may lead in many cases to inefficient and computationally costly schemes.

On the other hand, explicit expressions are more desirable, as provided by Pan [11] for the fundamental solution of MEE materials and by Buroni and Sáez [12] for its derivatives. In this approach, one needs to solve an eigenproblem for each evaluation point, and one deals with the typical problem of mathematical degeneracy which can arise in explicit formulations. Buroni and Sáez [12] have presented a set of explicit solutions, both for the fundamental solution and its derivatives, which consider all possible kind of degeneracies - however one needs to know which solution to apply, and this is not a trivial task. In addition to the above-mentioned contributions, additional effort has been invested in recent years for constructing adequate explicit solutions. For instance, see the works of Xie et al. [13] and [14] for $\mathrm{PE}$ and MEE materials, respectively, where both solutions degenerate mathematically. It is remarkable the explicit approach recently proposed by Xie et al. [15] for PE materials, which is valid for degenerate cases. However, to the authors' best knowledge no non-degenerate explicit fundamental solution has been derived for MEE materials. A solution constructed by Radon-Stroh for- 
malism [10, 17] has been proposed and two different evaluation approaches, complex form and real form, have been suggested and discussed in $\mathrm{Hsu}$ et al. [16]. Between these two approaches, the one based upon the complex form also has the feature of single time evaluation, a concept similar to the presented in this study.

Moreover, some approximate approaches such as the data base and interpolation scheme proposed by Wilson \& Cruse [18] have also been proposed. See for instance the work by Muraishi [19] for PE materials. Another interesting approach based on the relationship between the Rayleigh expansion and Fourier representation, which leads to a series representation in terms of spherical harmonics has been proposed recently [20]. In the context of contact and crack problems see the work by Fabrikant [21] and for thermo-MEE fundamental solutions the work by Pasternak et al. [22].

In summary, existing fundamental solutions exhibit at least one of the following inconveniences: $(i)$ they are implicit, or $(i i)$ they are mathematically degenerated, preventing the use of such solutions in the general case, and/or ( iii) they are evaluated by an approximated procedure. In this context, the aim of this work is to provide a numerical scheme suitable for numerical implementation of the 3D fundamental solution and its first- and second-order derivatives for PE, PM and MEE materials with general anisotropy. The remainder of the paper is organized as follows: In Section 2, basic equations and notation for magnetoelectroelasticity are introduced. In order to overcome the above mentioned limitations, in Section 3 a new expression for the fundamental solution in MEE materials is presented which has three relevant characteristics: $(i)$ it is explicit in terms of the Stroh's eigenvalues, $(i i)$ it remains well-defined when some Stroh's eigenvalues are equal (mathematical degeneracy) or nearly equal (quasi-mathematical degeneracy), and ( $i i i$ ) it is exact. Next, by using this new expression and a representation of the solution based on double Fourier series, a very fast and robust numerical scheme for the evaluation of the 3D fundamental solution and its derivatives is presented

in Section 4. Some numerical results validate the proposed approach while showcasing the attained accuracy. We discuss the conclusions in Section 5.

\section{Basic equations of linear magnetoelectroelasticity}

Let $x_{i}(i=1,2,3)$ be a Cartesian coordinate system in three-dimensions. The extended notation introduced by Barnett \& Lothe [23] for PE materials is very convenient for the purpose of this work. In this way, the linear MEE 
problem can be formulated in an elastic-like fashion by extending the elastic displacement field vector $u_{i}$ with the addition of the electric potential $\varphi$ and the magnetic potential $\vartheta$ as [24]

$$
u_{J}= \begin{cases}u_{j} & J \leqslant 3 \\ \varphi & J=4 \\ \vartheta & J=5\end{cases}
$$

and by defining an extended elasticity tensor with the following components $[24]$

$$
C_{i J K m}= \begin{cases}c_{i j k m} & J, K \leqslant 3 \\ e_{m i j} & J \leqslant 3 ; K=4 \\ e_{i k m} & J=4 ; K \leqslant 3 \\ q_{m i j} & J \leqslant 3 ; K=5 \\ q_{i k m} & J=5 ; K \leqslant 3 \\ -\lambda_{i m} & J=4 ; K=5 \text { or } J=5 ; K=4 \\ -\epsilon_{i m} & J, K=4 \\ -\mu_{i m} & J, K=5 .\end{cases}
$$

where $c_{i j k l}, \epsilon_{i l}$ and $\mu_{i l}$ denote the components of the elastic stiffness tensor at constant electric and magnetic fields, the dielectric permittivity tensor at constant strains and magnetic fields, and the magnetic permeabilities tensor at constant stresses and electric displacements, respectively; $e_{i j k}, q_{i j k}$ and $\lambda_{i l}$ are the PE coupling coefficients at constant magnetic fields, PM coupling coefficients at constant electric fields and ME coupling coefficients at constant strains and electric fields, respectively. We assume isothermal conditions. The material constants tensors show the following symmetry conditions

$$
\begin{gathered}
c_{i j k l}=c_{j i k l}=c_{i j l k}=c_{k l i j}, \quad e_{k i j}=e_{k j i}, \quad q_{k i j}=q_{k j i}, \\
\epsilon_{k l}=\epsilon_{l k}, \quad \lambda_{k l}=\lambda_{l k}, \quad \mu_{k l}=\mu_{l k} .
\end{gathered}
$$

Due to these symmetries, $C_{i J K m}=C_{m K J i}$ is satisfied. Moreover, the elastic constant, dielectric permittivity, magnetic permeability and ME coupling tensors are positive definite, i.e.

$$
\begin{gathered}
c_{i j k m} \gamma_{i j} \gamma_{k m}>0, \epsilon_{i j} E_{i} E_{j}>0, \mu_{i j} H_{i} H_{j}>0, \lambda_{i m} E_{i} H_{j}>0 \\
\forall \gamma_{k m}, E_{j}, H_{j} \in \mathbb{R} ; \gamma_{k m}=\gamma_{m k} \neq 0, E_{j} \neq 0, H_{j} \neq 0 .
\end{gathered}
$$


and no constraint is imposed on the PE and PM coupling tensors. Equation (4) is known as the strong convexity condition and it is equivalent to positive definiteness of the internal energy function.

In the definitions above, the lowercase (elastic) and uppercase (extended) subscripts take values 1, 2, 3 and 1, 2, 3 (elastic), 4 (electric), 5 (magnetic), respectively. As pointed out by Fan [25] for piezoelectricity, these matrix representations are not tensors. So one has to be careful when changing coordinates systems (as the ones used in Section 4). Then, using the introduced matrix representation, elliptic equilibrium equations for the elastic, electric and magnetic problems in terms of the extended displacements can be recast in a similar way to Navier's equation of elasticity as

$$
C_{i J K m} u_{K, m i}+f_{J}=0
$$

where $f_{J}$ is the extended body force vector, defined as

$$
f_{J}= \begin{cases}f_{j} & J \leqslant 3 \\ -f^{e} & J=4 \\ -f^{m} & J=5,\end{cases}
$$

being $f_{i}, f^{e}$ and $f^{m}$ the three components of body forces, the electric charge density and the electric current density, respectively. As usual, comma denotes differentiation. Note that uncoupled problems, i.e., purely elastic, electric and/or magnetic, can be considered by setting the corresponding coefficients $e_{i j k}, q_{i j k}$ and/or $\lambda_{i l}$ to zero.

\section{Non-degenerate fundamental solution}

The fundamental solution is defined as a two-point second-order tensor in a five-dimension space with components $U_{K P}$ such that satisfies the elliptic partial differential equations (5) where the generalised body force vector corresponds to a point load $f_{J}=\delta_{J P} \delta\left(\mathbf{x}-\mathbf{x}^{\prime}\right)$ being $\delta\left(\mathbf{x}-\mathbf{x}^{\prime}\right)$ the Dirac delta function located at the source point $\mathbf{x}^{\prime}$ and $\delta_{J K}$ the five-dimension Kronecker delta. In homogeneous media the fundamental solutions depends on the relative vector $\mathbf{x}-\mathbf{x}^{\prime}$ so, for simplicity it is considered that the Cartesian coordinate system has the origin at the source point $\mathbf{x}^{\prime}$, thus the fundamental solution is just a function of the evaluation point $\mathbf{x}$. For a physical interpretation see [12]. 
The fundamental solution can be expressed as a singular term by a modulation function $\mathbf{H}$ as

$$
U_{J K}(\mathbf{x})=\frac{1}{4 \pi r} H_{J K}(\mathbf{x})
$$

where $\mathbf{x}=r$ ê with $r=|\mathbf{x}| \neq 0$. The modulation function $H_{J K}(\mathbf{x})$ depends on the direction of $\mathbf{x}$ but not on its modulus, so $H_{J K}(\mathbf{x})=H_{J K}(\hat{\mathbf{e}})$. This function can be put in the context of the Stroh formalism [26] being known as one of the three extended Barnett-Lothe tensors, which is symmetric and $\mathbf{H}(\hat{\mathbf{e}})=\mathbf{H}(-\hat{\mathbf{e}})$. Hence, $\mathbf{U}(\mathbf{x})$ is also symmetric and even, i.e.:

$$
U_{J K}(\mathbf{x})=U_{J K}(-\mathbf{x}) .
$$

Therefore, the following parity relationships for the derivatives of $\mathbf{U}(\mathbf{x})$

$$
U_{J K, m}(\mathbf{x})=-U_{J K, m}(-\mathbf{x})
$$

and

$$
U_{J K, m n}(\mathbf{x})=U_{J K, m n}(-\mathbf{x})
$$

are satisfied.

The tensor $H_{J K}$ can be evaluated as $[12,27]$

$$
H_{J K}(\hat{\mathbf{e}})=\frac{1}{\pi} \int_{-\infty}^{+\infty} \Gamma_{J K}^{-1}(p) d p
$$

with

$$
\Gamma_{J K}(p)=Q_{J K}+\left(R_{J K}+R_{K J}\right) p+T_{J K} p^{2},
$$

and

$$
Q_{J K}=C_{i J K m} n_{i} n_{m}, \quad R_{J K}=C_{i J K m} n_{i} m_{m}, \quad T_{J K}=C_{i J K m} m_{i} m_{m},
$$

where $n_{i}$ and $m_{i}$ are the components of any two mutually orthogonal unit vectors such that $(\mathbf{n}, \mathbf{m}, \hat{\mathbf{e}})$ is a right-handed triad. Note that $Q_{J K}$ and $T_{J K}$ are symmetric like their elastic counterparts [26], but the MEE coupling cause the loss of positive definiteness of these matrices. However, as Ting [26] shows for piezoelectric materials, it can be proven that $Q_{J K}, T_{J K}$ and $\Gamma_{J K}$ are non-singular in this case, so their inverses are guaranteed. Moreover, $\mathbf{H}$ and $\mathbf{U}$ are independent of the choice of the unit vectors $\mathbf{m}$ and $\mathbf{n}$ on the oblique plane. 
The kernel in equation (11) is a single-valued holomorphic function in the upper complex half-plane except at the five complex poles with positive imaginary part and their conjugates that corresponds to the roots of the ten-order polynomial equation

$$
|\boldsymbol{\Gamma}(p)|=0
$$

The determinant in (14) can be factorised as

$$
|\boldsymbol{\Gamma}(p)|=|\mathbf{T}| \prod_{\xi=1}^{5}\left(p-p_{\xi}\right)\left(p-\bar{p}_{\xi}\right),
$$

where $p_{\xi}$ are known as the Stroh's eigenvalues and the bar over $p_{\xi}$ denotes the complex conjugate and T is defined in equation (13). Stroh's eigenvalues can be obtained as the roots of tenth-order characteristic equation (14) as well as solving the eigen-problem, as described in Appendix A. Then, assuming that all Stroh's eigenvalues are different, the integration in equation (11) can be done by the Cauchy's residue theory to yield

$$
H_{J K}(\hat{\mathbf{e}})=\frac{2 \mathrm{i}}{|\mathbf{T}|} \sum_{\alpha=1}^{5} \frac{\hat{\Gamma}_{J K}\left(p_{\alpha}\right)}{\left(p_{\alpha}-\bar{p}_{\alpha}\right) \prod_{\substack{\xi=1 \\ \xi \neq \alpha}}^{5}\left(p_{\alpha}-p_{\xi}\right)\left(p_{\alpha}-\bar{p}_{\xi}\right)},
$$

where $\hat{\Gamma}_{J K}$ is the adjugate of $\Gamma_{J K}$ defined as $\Gamma_{P J}(p) \hat{\Gamma}_{J K}(p)=|\boldsymbol{\Gamma}(p)| \delta_{P K}$ and $\mathrm{i}=\sqrt{-1}$. Clearly, this expression is not valid for degenerate cases when there are repeated Stroh's eigenvalues. Following an idea of [27], equation (16) can be algebraically modified in order to obtain a well-defined solution, valid even for repeated Stroh's eigenvalues of any multiplicity, as explained below.

The adjugate matrix $\hat{\Gamma}_{J K}(p)$ is a polynomial in $p$ of degree eight. Let

$$
\hat{\Gamma}_{J K}(p)=\sum_{n=0}^{8} p^{n} \hat{\Gamma}_{J K}^{(n)},
$$

where $\hat{\Gamma}_{J K}^{(n)}(n=0, \ldots, 8)$ are real symmetric matrices which only depend on the material properties and the position vector ê. These matrices can be computed in a straightforward way in terms of the Stroh matrices (13) 
through the co-factor matrix of $\Gamma_{J K}(p)$ (12). Shiah et al. [29] present explicit formulas for the elastic counterpart. However for the MEE case, the co-factor matrix involves computation of determinants of $4 \times 4$ matrices -instead of $2 \times 2$ matrices - which leads to expressions too large to be written here. Then, the tensor $H_{J K}$ in equation (16) can be rewritten as

$$
H_{J K}(\hat{\mathbf{e}})=\frac{1}{|\mathbf{T}|} \sum_{n=0}^{8} q_{n} \hat{\Gamma}_{J K}^{(n)},
$$

with

$$
q_{n}=\sum_{\alpha=1}^{5} \frac{2 \mathrm{i} p_{\alpha}^{n}}{\left(p_{\alpha}-\bar{p}_{\alpha}\right) \prod_{\substack{\xi=1 \\ \xi \neq \alpha}}^{5}\left(p_{\alpha}-p_{\xi}\right)\left(p_{\alpha}-\bar{p}_{\xi}\right)} .
$$

Since $H_{J K}$ and $\hat{\Gamma}_{J K}^{(n)}$ are real, $q_{n}: \mathbb{C}^{10} \rightarrow \mathbb{R}$. Note that this last expression for $q_{n}$ is not valid for degenerate cases. However, there is no physical reason for that and the indetermination can be removed. In fact, equation (19) can be factorised such that the common denominator for all $q_{n}(n=0, \ldots, 8)$ is $\prod_{\alpha=1}^{5} \prod_{\xi=1}^{5}\left(p_{\alpha}-\bar{p}_{\xi}\right)$. This denominator is always different from zero, so $q_{n}$ functions remain well-defined even in degenerate cases. The corresponding explicit expressions for the numerator of $q_{n}(n=0, \ldots, 8)$ are quite large to be shown herein. However, the factorization can be straightforwardly obtained with any software of symbolic computation as MATHEMATICA [28].

At this point, it is worth to remind that previous formulas with the same structure of eq. (16) fail when used for mathematical degenerated cases, i.e., when some repeated Stroh's eigenvalues exist. Even more important, numerical instabilities are observed in quasi-degenerated cases when Stroh's eigenvalues are sufficiently close. Such situation may happen depending both on the material properties $C_{i J K m}$ and the direction of the vector $\hat{\mathbf{e}}$. The formulation presented by Buroni and Sáez [12] overcame such degeneracy by using a set of solutions depending on the kind of degeneracy. The advantage of expression (18) combined with the factorised coefficients (19) consists in both being valid independently from the kind of degeneracy involved, as opposed to previous approaches. In order to illustrate this, consider a transversely isotropic piezoelectric material (Material A) where the constants are summarised in Table B.5 of the Appendix B. For this material symmetry, when 


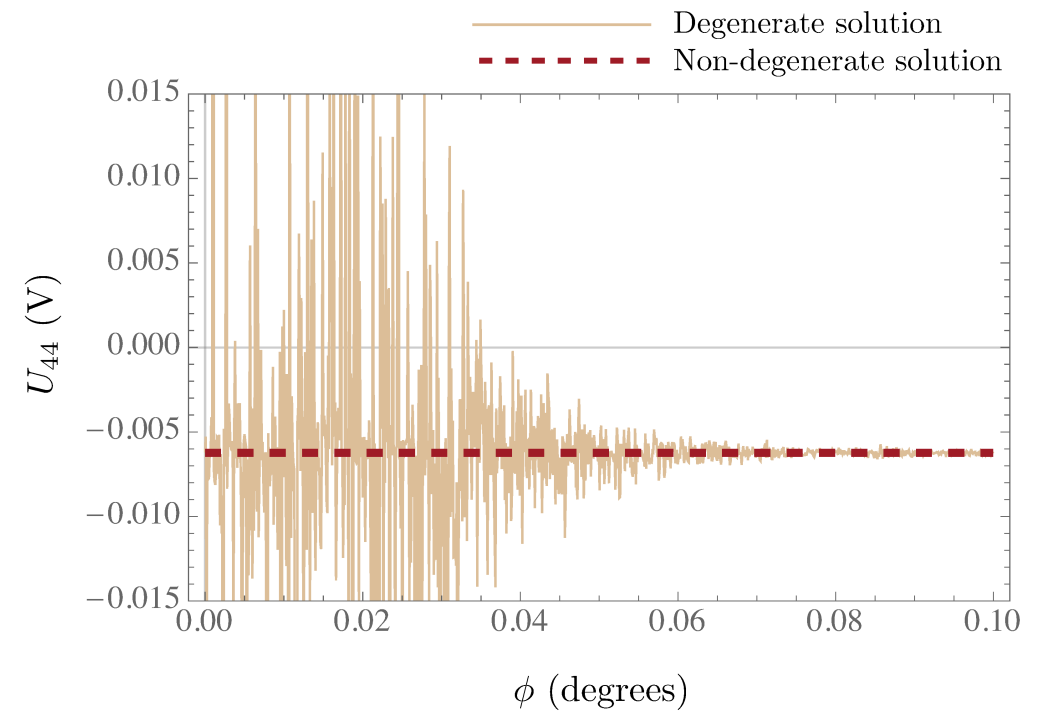

Figure 1: $U_{44}$ component versus $\phi$-angle for piezoelectric Material A $(|\mathbf{x}|=1)$.

source and field points are both along the $x_{3}$-axis, the four Stroh's eigenvalues associated to the piezoelectric problem become equal to $i=\sqrt{-1}$. Figure 1 shows the evolution of component $U_{44}$ versus the angle $\phi$ defined as the angle between the $x_{3}$-axis and the the position vector $\mathbf{x}$. The modulus of the position vector is $|\mathbf{x}|=1$. The degenerate solution (7) and (16) are plotted with a continuous line. For angles around $\phi \approx 0.1$ the solution slowly starts to degrade and for $0<\phi \lesssim 0.07^{\circ}$, Stroh's eigenvalues are almost coincident resulting in a numerically unstable solution due to this quasi-degeneracy, as shown in the figure. As mentioned earlier, for $\phi=0^{\circ}$ there is the degeneracy $p_{i}=\sqrt{-1}$ for all $i(i=1 \ldots 4)$, so expression (16) is not defined. The new solution is drawn with a dashed line, which is smooth, accurate and well-defined everywhere. Similar behaviour is obtained for other components.

Table 1 shows a compararison of the components $U_{J K}$ obtained with the present formulation and with the formulation by Buroni and Sáez [12] as a reference solution for a MEE case. The material is a transversely isotropic MEE (Material B) with properties summarized in Table B.6 of Appendix B and the evaluation point is $\mathbf{x}=(1,1,-1)^{T}$. The relative error is computed as the difference with respect to the reference solution normalized by the reference solution. The computations throughout the paper were performed using double precision, 64 bit floating point. Also no unsafe optimizations were used in compiled Fortran routines, to comply with IEEE-754 standard. 


\begin{tabular}{cccc}
\hline$(J K)$ & Buroni \& Sáez $(2010)$ & present work & relative error \\
\hline 11 & $7.7141369757490015 \times 10^{-4}$ & $7.7141366651463308 \times 10^{-4}$ & $4.0264 \times 10^{-8}$ \\
12 & $1.2027080088980113 \times 10^{-4}$ & $1.2027080690932257 \times 10^{-4}$ & $5.0050 \times 10^{-8}$ \\
13 & $1.2945920760425760 \times 10^{-4}$ & $1.2945919822994514 \times 10^{-4}$ & $7.2411 \times 10^{-8}$ \\
14 & $1.0347227658113671 \times 10^{-4}$ & $1.0347226896648796 \times 10^{-4}$ & $7.3591 \times 10^{-8}$ \\
15 & $-4.2667071176276332 \times 10^{-6}$ & $-4.2667067164579118 \times 10^{-6}$ & $9.4023 \times 10^{-8}$ \\
22 & $7.7141369757490015 \times 10^{-4}$ & $7.7141366651463308 \times 10^{-4}$ & $4.0264 \times 10^{-8}$ \\
23 & $1.2945920760425762 \times 10^{-4}$ & $1.2945919822994529 \times 10^{-4}$ & $7.2411 \times 10^{-8}$ \\
24 & $1.0347227658113787 \times 10^{-4}$ & $1.0347226896648809 \times 10^{-4}$ & $7.3591 \times 10^{-8}$ \\
25 & $-4.2667071176276350 \times 10^{-6}$ & $-4.2667067164579153 \times 10^{-6}$ & $9.4023 \times 10^{-8}$ \\
33 & $4.8476833195420852 \times 10^{-4}$ & $4.8476831665570996 \times 10^{-4}$ & $3.1558 \times 10^{-8}$ \\
34 & $5.0073977011499986 \times 10^{-4}$ & $5.0073975331344647 \times 10^{-4}$ & $3.3553 \times 10^{-8}$ \\
35 & $2.4195754528575155 \times 10^{-5}$ & $2.4195754183322036 \times 10^{-5}$ & $1.4269 \times 10^{-8}$ \\
44 & $-3.4041791147399341 \times 10^{-3}$ & $-3.4041790013154500 \times 10^{-3}$ & $3.3319 \times 10^{-8}$ \\
45 & $2.9749048777373694 \times 10^{-5}$ & $2.9749048155802629 \times 10^{-5}$ & $2.0894 \times 10^{-8}$ \\
55 & $-4.1918947944612555 \times 10^{-6}$ & $-4.1918946641014401 \times 10^{-6}$ & $3.1098 \times 10^{-8}$ \\
\hline avg & & & $5.1688 \times 10^{-8}$ \\
\hline
\end{tabular}

Table 1: Components $U_{J K}$ for material B when the evaluation point is $\mathbf{x}=(1,1,-1)^{T}$.

Very recently, an interesting work by Xie et al. [15] has proposed an alternative unified formula for anisotropic piezoelectric fundamental solution and its first-derivative which could be also extended to MEE materials. After an algebraic rearrangement and using recursive relations, the integral coefficients (equivalent to $q_{n}$ before integration) are calculated by the Cauchy residue theorem. The resulting expressions in explicit-form seem to be more compact than the ones presented herein, but they are also more labour-intensive to implement. The extension to MEE materials seems to involve even more cumbersome manipulating algebra. In any case, the underlying principle that permits to remove the mathematical degeneracy is the factorization of the denominator in eq. (16), since this comes from the mathematical structure and not from physical arguments. For these reasons, several factorizations could be proposed in order to speed up the numerical implementation. However, in order to ensure an efficient integration scheme in boundary integral methodologies we adopt the approach presented in next section.

\section{Fast Fourier series approach}

As mentioned, equation (7) shows that the displacement fundamental solution can be expressed via separation of variables as a singular function depending only on the radial distance $r$, and the regular modulation function $H_{J K}(\hat{\mathbf{e}})$ which depends only on $\hat{\mathbf{e}}$. This vector can be expressed in terms of 
spherical coordinates $\theta$ and $\phi$ as $\hat{\mathbf{e}}=(\sin \phi \cos \theta, \sin \phi \sin \theta, \cos \phi)(-\pi \leq$ $\theta<\pi, 0 \leq \phi<\pi)$, and therefore the Barnett-Lothe tensor $H_{J K}(\theta, \phi)$ can be written as a periodic function in both $\theta$ and $\phi$ with a period of $2 \pi$ and $\pi$, respectively. Due to this periodic nature, $H_{J K}(\theta, \phi)$ admits a double Fourier series representation which can be expressed in a compact form in terms of complex exponentials [29], by generalizing the ideas previously presented for the purely elastic case, as

$$
H_{J K}(\theta, \phi)=\sum_{m=-\infty}^{\infty} \sum_{n=-\infty}^{\infty} \lambda_{J K}^{(m, n)} \mathrm{e}^{\mathrm{i}(m \theta+\rho n \phi)},
$$

being $\rho=2$ and the Fourier expansion coefficients given by

$$
\lambda_{J K}^{(m, n)}=\frac{1}{2 \pi^{2}} \int_{-\frac{\pi}{\rho}}^{\frac{\pi}{\rho}} \int_{-\pi}^{\pi} H_{J K}(\theta, \phi) \mathrm{e}^{-\mathrm{i}(m \theta+\rho n \phi)} \mathrm{d} \theta \mathrm{d} \phi,
$$

where the integrations involved can be calculated numerically by standard Gaussian quadrature. One can also note that the double Fourier series representation (20) for the displacement solution $H_{J K}(\theta, \phi)$ converges absolutely and uniformly since $\frac{\partial^{2} H_{J K}}{\partial \theta \partial \phi}$ exists and is continuous. Then, the $H_{J K}(\theta, \phi)$ can be approximated by a Fourier polynomial by truncating the infinite series (20) in a sufficiently large number of terms $\alpha$ to yield results as accurate as required, thus

$$
H_{J K}(\theta, \phi)=\sum_{m=-\alpha}^{\alpha} \sum_{n=-\alpha}^{\alpha} \lambda_{J K}^{(m, n)} \mathrm{e}^{\mathrm{i}(m \theta+\rho n \phi)} .
$$

The key point of the double Fourier polynomial representation (22) is that, once the Fourier coefficients are known, each term of the solution becomes a simple complex exponential function with respect to the position angles $\theta$ and $\phi$ [29]. This is particularly useful for the evaluation of the Barnett-Lothe tensor $H_{J K}(\theta, \phi)$, as well as its derivatives, as shown below. On the other hand, the previously obtained explicit and non-degenerate expression (18) for $H_{J K}(\theta, \phi)$ can be used in order to compute the Fourier expansion coefficients (21), without any restrictions due to possible mathematical degeneracies.

Following Tan et al. [30], further improvements in efficiency can be performed. The Fourier coefficients $\lambda_{J K}^{(m, n)}$ can be separated into the real and imaginary part as

$$
\lambda_{J K}^{(m, n)}=\mathcal{R}_{J K}^{(m, n)}+\mathrm{i} \mathcal{I}_{J K}^{(m, n)} .
$$


Since $H_{J K}(\theta, \phi) \in \mathbb{R}$, equation (22) is rewritten as

$$
H_{J K}(\theta, \phi)=\sum_{m=-\alpha}^{\alpha} \sum_{n=-\alpha}^{\alpha} h_{J K}^{(m, n)}(\theta, \phi),
$$

where

$$
h_{J K}^{(m, n)}(\theta, \phi):=\mathcal{R}_{J K}^{(m, n)} \cos (m \theta+\rho n \phi)-\mathcal{I}_{J K}^{(m, n)} \sin (m \theta+\rho n \phi) .
$$

By noting that $\lambda_{J K}^{(m, n)}$ and $\lambda_{J K}^{(-m,-n)}$ are complex conjugate, some terms in (24) are equals. Therefore, the sum in (24) can be reduced to yield [30]

$$
\begin{aligned}
H_{J K}(\theta, \phi) & =2 \sum_{m=1}^{\alpha} \sum_{n=1}^{\alpha} h_{J K}^{(m, n)}(\theta, \phi)+2 \sum_{m=1}^{\alpha} \sum_{n=-\alpha}^{-1} h_{J K}^{(m, n)}(\theta, \phi) \\
& +2 \sum_{m=1}^{\alpha} h_{J K}^{(m, 0)}(\theta, \phi)+2 \sum_{n=1}^{\alpha} h_{J K}^{(0, n)}(\theta, \phi)+\mathcal{R}_{J K}^{(0,0)} .
\end{aligned}
$$

Note that for real and even functions the imaginary part of the Fourier coefficients is zero. Although $H_{J K}(\hat{\mathbf{e}})$ is an even function in $\hat{\mathbf{e}}$ (see equation $(8)$ ), it is not in $\theta$ and $\phi$, leading to the fact that the imaginary part of the Fourier coefficients $\mathcal{I}_{J K}^{(m, n)}$ are, in general, non zero in the present formulation. However, it is important to remark that the Fourier polynomial representation (26) of $H_{J K}(\theta, \phi)$ is real-valued.

It should be noted that the implementation of the Barnett-Lothe's formula used to compute the Fourier coefficients, previous formulations [29, 30] have selected a period of $2 \pi$ for $\phi$ variable [31] for practical reasons. Those formulations are essentially the same to the one presented in this work setting $\rho=1$ in the expressions above. The difference between adopting one or other period, i.e., taking $\rho=1$ or $\rho=2$, is addressed length below.

In the same way as for the fundamental solution, its first-order derivative may be expressed as a singular part by a modulation function which only depends on $\hat{\mathbf{e}}$ as

$$
U_{J K, l}(\mathbf{x})=\frac{1}{4 \pi r^{2}} \tilde{U}_{J K l}(\hat{\mathbf{e}})
$$

being the modulation function given by

$$
\tilde{U}_{J K l}(\hat{\mathbf{e}})=-\hat{e}_{l} H_{J K}+\frac{m_{l}}{\sin \phi} \frac{\partial H_{J K}}{\partial \theta}+n_{l} \frac{\partial H_{J K}}{\partial \phi}
$$


where $n_{l}$ and $m_{l}$ are the components of the two orthogonal vectors used in (13) and taken as $\mathbf{n}=(\cos \phi \cos \theta, \cos \phi \sin \theta,-\sin \phi)$ and $\mathbf{m}=(-\sin \theta$, $\cos \theta, 0)$; so $\hat{e}_{l}$ is the $l$-component of $\hat{\mathbf{e}}=(\sin \phi \cos \theta, \sin \phi \sin \theta, \cos \phi)$.

Furthermore, the second-order derivative of the fundamental solution may be written as

$$
U_{J K, l s}(\mathbf{x})=\frac{1}{4 \pi r^{3}} \tilde{U}_{J K l s}(\hat{\mathbf{e}}),
$$

where the modulation function $\tilde{U}_{J K l s}$ is given by

$$
\begin{aligned}
\tilde{U}_{J K l s}(\hat{\mathbf{e}}) & =\hat{e}_{l}\left(2 \hat{e}_{s} H_{J K}-\frac{\partial H_{J K}}{\partial \phi} n_{l}\right)+\frac{\partial^{2} H_{J K}}{\partial \phi^{2}} n_{l} n_{s}-H_{J K}\left(m_{l} m_{s}+n_{l} n_{s}\right) \\
& +\frac{\partial H_{J K}}{\partial \phi}\left(\frac{\partial n_{l}}{\partial \phi} n_{s}-\hat{e}_{s}\left(n_{l}+n_{s}\right)\right) \\
& +\csc \phi\left(\frac{\partial H_{J K}}{\partial \phi} \frac{\partial n_{l}}{\partial \theta} m_{s}-\frac{\partial H_{J K}}{\partial \theta}\left(\hat{e}_{l} m_{l}+\hat{e}_{s}\left(m_{l}+m_{s}\right)\right)\right. \\
& +\frac{\partial^{2} H_{J K}}{\partial \theta \partial \phi}\left(m_{s} n_{l}+m_{l} n_{s}\right)-\frac{\partial H_{J K}}{\partial \theta} m_{l} n_{s} \cot \phi \\
& \left.+\left(\frac{\partial H_{J K}}{\partial \theta} \frac{d m_{l}}{d \theta}+\frac{\partial^{2} H_{J K}}{\partial \theta^{2}} m_{l}\right) m_{s} \csc \phi\right)
\end{aligned}
$$

In order to compute the derivatives of the fundamental solution (27) and (29), it is only required the corresponding derivatives of $H_{J K}(\theta, \phi)$ with respect to the spherical coordinates $\theta$ and $\phi$. If equation (26) is considered, these derivatives involve only trivial derivatives of trigonometric functions in $h_{J K}^{(m, n)}(\theta, \phi)$ according to $(25)$. Therefore, it is emphasized that $\mathcal{R}_{J K}^{(m, n)}$ and $\mathcal{I}_{J K}^{(m, n)}$ are not function of the position vector $\hat{\mathbf{e}}$, and only need to be computed once for a given material. This is the main advantage of the proposed approach.

In equations (28) and (30) there are removable singularities when $\phi=0$. This difficulty can be easily overcomed with, for instance, a rotation of the coordinate system $x_{i}(i=1,2,3)$ on the $x_{2}$-axis by an angle of $\frac{\pi}{2}$ represented by the following proper orthogonal transformation matrix

$$
\boldsymbol{\Omega}^{\left.(2)\right|_{\frac{\pi}{2}}}=\left(\begin{array}{ccc}
0 & 0 & -1 \\
0 & 1 & 0 \\
1 & 0 & 0
\end{array}\right) .
$$


Then, points on the $x_{3}$-axis such as $\hat{\mathbf{e}}_{(3)}=(0,0,1)^{T}$ or $\hat{\mathbf{e}}_{(3)}=(0,0,-1)^{T}$, in the new coordinate system $x_{i}^{*}(i=1,2,3)$ they become $\hat{\mathbf{e}}_{(3)}^{*}=(-1,0,0)^{T}$ or $\hat{\mathbf{e}}_{(3)}^{*}=(1,0,0)^{T}$, respectively. This artifice can be used since the expressions in equations (28) and (30) are well-defined for vectors like $\hat{\mathbf{e}}_{(3)}^{*}$. Modulation functions $\tilde{U}_{I K j}$ and $\tilde{U}_{I K j l}$ are not tensors. They can be transformed according to the following rules

$$
\tilde{U}_{I K j}\left(\hat{\mathbf{e}}_{(3)}\right)=\Omega_{A I}^{\left.(2)\right|_{\frac{\pi}{2}}} \Omega_{B K}^{\left.(2)\right|_{\frac{\pi}{2}}} \Omega_{c j}^{(2) \mid \frac{\pi}{2}} \tilde{U}_{A B c}^{*}\left(\hat{\mathbf{e}}_{(3)}^{*}\right),
$$

and

$$
\tilde{U}_{I K j l}\left(\hat{\mathbf{e}}_{(3)}\right)=\Omega_{A I}^{(2) \mid \frac{\pi}{2}} \Omega_{B K}^{(2) \mid \frac{\pi}{2}} \Omega_{c j}^{(2) \mid \frac{\pi}{2}} \Omega_{d l}^{(2) \mid \frac{\pi}{2}} \tilde{U}_{A B c d}^{*}\left(\hat{\mathbf{e}}_{(3)}^{*}\right) .
$$

Naturally, since $\boldsymbol{\Omega}^{(2) \mid \frac{\pi}{2}}$ is not necessarily a material symmetry operation, the MEE properties must be expressed in the $x_{i}^{*}(i=1,2,3)$ system as

$$
\begin{aligned}
c_{i j k l}^{*} & =\Omega_{i a}^{(2) \mid \frac{\pi}{2}} \Omega_{j b}^{(2) \mid \frac{\pi}{2}} \Omega_{k c}^{(2) \mid \frac{\pi}{2}} \Omega_{l d}^{(2) \mid \frac{\pi}{2}} c_{a b c d}, \\
e_{i j k}^{*} & =\Omega_{i a}^{(2) \mid \frac{\pi}{2}} \Omega_{j b}^{(2) \mid \frac{\pi}{2}} \Omega_{k c}^{(2) \mid \frac{\pi}{2}} e_{a b c}, \\
q_{i j k}^{*} & =\Omega_{i a}^{(2) \mid \frac{\pi}{2}} \Omega_{j b}^{(2) \mid \frac{\pi}{2}} \Omega_{k c}^{(2) \mid \frac{\pi}{2}} q_{a b c}, \\
\epsilon_{i j}^{*} & =\Omega_{i a}^{(2) \mid \frac{\pi}{2}} \Omega_{j b}^{(2) \mid \frac{\pi}{2}} \epsilon_{a b}, \\
\lambda_{i j}^{*} & =\Omega_{i a}^{(2) \mid \frac{\pi}{2}} \Omega_{j b}^{(2) \mid \frac{\pi}{2}} \lambda_{a b}, \\
\mu_{i j}^{*} & =\Omega_{i a}^{(2) \mid \frac{\pi}{2}} \Omega_{j b}^{(2) \mid \frac{\pi}{2}} \mu_{a b},
\end{aligned}
$$

in order to compute the solutions in the $x_{i}^{*}(i=1,2,3)$ coordinate system.

Table 2 compares the fundamental solution for fully MEE Material $\mathrm{C}$ (properties listed in table B.7) at point $\mathbf{x}=(1,1,1)^{T}$ obtained with the Fourier series approach, the non-degenerate approach and the solution by Buroni \& Sáez [12] as reference. Table 3 presents the results obtained with the proposed formulation and its comparison with the results by Buroni \& Sáez and finite difference approach for the $U_{J K, 3}$ derivatives at point $\mathbf{x}=(1,1,1)^{T}$ [12]. Table 4 presents the compararison for the $U_{J K, 12}$ derivatives at the same point with the finite difference approach by using the Buroni \& Sáez solution [12]. For all cases, 256 Gauss points have been used for integration of the coefficients (21), and the series take 20 terms. Very good agreement is observed amongst the solutions for all cases. 


\begin{tabular}{cccr}
\hline$(J K)$ & Buroni \& Sáez $(2010)$ & present work $($ non degenerate) & present work $($ Fourier Series $\rho=2)$ \\
\hline 11 & $8.7225398002742391 \times 10^{-4}$ & $8.7225310879278819 \times 10^{-4}$ & $8.7225302692569269 \times 10^{-4}$ \\
12 & $6.4154620180000892 \times 10^{-5}$ & $6.4154582776641815 \times 10^{-5}$ & $6.4154583909996026 \times 10^{-5}$ \\
13 & $3.1446825949164037 \times 10^{-4}$ & $3.1446793068922219 \times 10^{-4}$ & $3.1446789927188727 \times 10^{-4}$ \\
14 & $-1.2680519416303164 \times 10^{-3}$ & $-1.2680506415118376 \times 10^{-3}$ & $-1.2680505211279109 \times 10^{-3}$ \\
15 & $-5.1231568254934725 \times 10^{-7}$ & $-5.1231513273905938 \times 10^{-7}$ & $-5.1231508865968785 \times 10^{-7}$ \\
22 & $7.6090426614139801 \times 10^{-4}$ & $7.6090350460375387 \times 10^{-4}$ & $7.6090344129206722 \times 10^{-4}$ \\
23 & $4.9374127465890965 \times 10^{-5}$ & $4.9374095746782780 \times 10^{-5}$ & $4.9374098591871851 \times 10^{-5}$ \\
24 & $4.5315107542192896 \times 10^{-4}$ & $4.5315058718214098 \times 10^{-4}$ & $4.5315053684158277 \times 10^{-4}$ \\
25 & $2.8343787079494569 \times 10^{-7}$ & $2.8343751170138947 \times 10^{-7}$ & $2.8343748212786792 \times 10^{-7}$ \\
33 & $1.0440331060742933 \times 10^{-3}$ & $1.0440321207110419 \times 10^{-3}$ & $1.0440320278586277 \times 10^{-3}$ \\
34 & $-1.8426611715379502 \times 10^{-3}$ & $-1.8426594309862083 \times 10^{-3}$ & $-1.8426592695127788 \times 10^{-3}$ \\
35 & $-6.5916283018091271 \times 10^{-7}$ & $-6.5916214947368479 \times 10^{-7}$ & $-6.5916209154751710 \times 10^{-7}$ \\
44 & $1.2157849166703620 \times 10^{-2}$ & $1.2157837419870272 \times 10^{-2}$ & $1.2157836354733913 \times 10^{-2}$ \\
45 & $1.8466901349619211 \times 10^{-6}$ & $1.8466881829979327 \times 10^{-6}$ & $1.8466880294716113 \times 10^{-6}$ \\
55 & $2.5182299673182738 \times 10^{-7}$ & $2.5182273648575246 \times 10^{-7}$ & $2.5182271523844513 \times 10^{-7}$ \\
\hline
\end{tabular}

Table 2: Green's function $U_{J K}$ at evaluation point $\mathbf{x}=(1,1,1)^{T}$ for MEE Material C.

\begin{tabular}{cccr}
\hline$(J K)$ & Finite difference & Buroni \& Sáez $(2010)$ & present work (Fourier Series $\rho=2)$ \\
\hline 11 & $-3.675418036705983 \times 10^{-13}$ & $-3.675418038147529 \times 10^{-13}$ & $-3.6754151848859640 \times 10^{-13}$ \\
12 & $-1.022473809428751 \times 10^{-13}$ & $-1.022473811350401 \times 10^{-13}$ & $-1.0224716811402825 \times 10^{-13}$ \\
13 & $-1.130914219850401 \times 10^{-14}$ & $-1.130914522684852 \times 10^{-14}$ & $-1.1309163083534299 \times 10^{-14}$ \\
14 & $3.207066889615237 \times 10^{-4}$ & $3.207067004117469 \times 10^{-4}$ & $3.2070743668963542 \times 10^{-4}$ \\
15 & $2.240137639332812 \times 10^{-7}$ & $2.240137681772090 \times 10^{-7}$ & $2.2403482815477269 \times 10^{-7}$ \\
22 & $-3.482702552773726 \times 10^{-13}$ & $-3.482702562831942 \times 10^{-13}$ & $-3.4827117098878988 \times 10^{-13}$ \\
23 & $2.457210719924869 \times 10^{-15}$ & $2.457208785075626 \times 10^{-15}$ & $2.4578986477356111 \times 10^{-15}$ \\
24 & $-1.386199675011690 \times 10^{-4}$ & $-1.386199583437589 \times 10^{-4}$ & $-1.3862246603200998 \times 10^{-4}$ \\
25 & $-1.123708543004768 \times 10^{-7}$ & $-1.123708508292927 \times 10^{-7}$ & $-1.1236385421649195 \times 10^{-7}$ \\
33 & $-8.404303395022170 \times 10^{-14}$ & $-8.404303328191928 \times 10^{-14}$ & $-8.4043404516964220 \times 10^{-14}$ \\
34 & $1.966253560794704 \times 10^{-4}$ & $1.966253482908411 \times 10^{-4}$ & $1.9662681422586747 \times 10^{-4}$ \\
35 & $1.850871822783363 \times 10^{-7}$ & $1.850871866296891 \times 10^{-7}$ & $1.8506905085345953 \times 10^{-7}$ \\
44 & $-3.346553299576044 \times 10^{6}$ & $-3.34655331275473 \times 10^{6}$ & $-3.3465596382245759 \times 10^{6}$ \\
45 & $-7.696119549791547 \times 10^{2}$ & $-7.69611965386665 \times 10^{2}$ & $-7.6960757074656776 \times 10^{2}$ \\
55 & $-1.323349050039724 \times 10^{2}$ & $-1.32334904512777 \times 10^{2}$ & $-1.3233341260687896 \times 10^{2}$ \\
\hline
\end{tabular}

Table 3: Derivative of Green's function $U_{J K, 3}$ at evaluation point $\mathbf{x}=(1,1,1)^{T}$ for MEE Material C.

In order to evaluate the performance of the double Fourier series approach, the following errors schemes are devised:

$$
e_{\mathrm{int}}^{(K, \alpha)}\left(\lambda_{I J}\right):=\frac{\sum_{m=-\alpha}^{\alpha} \sum_{n=-\alpha}^{\alpha}\left|\lambda_{I J}^{(m, n)}-\lambda_{I J}^{R(m, n)}\right|}{2 \alpha^{2}},
$$

where $\lambda_{I J}^{R(m, n)}$ is a reference matrix coefficient evaluated with a high number of Gauss abscissas, and $\lambda_{I J}^{(m, n)}$ is the same coefficient being evaluated with a 


\begin{tabular}{ccc}
\hline$(J K)$ & Finite difference with Buroni \& Sáez $(2010)$ & present work (Fourier Series $\rho=2)$ \\
\hline 11 & $1.7612993919238762 \times 10^{-4}$ & $1.7613069813436755 \times 10^{-4}$ \\
12 & $1.4372187047786456 \times 10^{-4}$ & $1.4372186057878247 \times 10^{-4}$ \\
13 & $-2.4864503755635603 \times 10^{-4}$ & $-2.4864148494725331 \times 10^{-4}$ \\
14 & $4.3863676517483414 \times 10^{-4}$ & $4.3861250202513018 \times 10^{-4}$ \\
15 & $-5.3289261534288785 \times 10^{-4}$ & $-5.3288733367096404 \times 10^{-4}$ \\
22 & $-3.8721191709814931 \times 10^{-4}$ & $-3.8719435901746122 \times 10^{-4}$ \\
23 & $-2.6527046054561354 \times 10^{-4}$ & $-2.6527176860501949 \times 10^{-4}$ \\
24 & $-6.1505992611293401 \times 10^{-4}$ & $-6.1506203927697429 \times 10^{-4}$ \\
25 & $3.6085826796324048 \times 10^{-4}$ & $3.6084569437355527 \times 10^{-4}$ \\
33 & $-5.5435380481296344 \times 10^{-8}$ & $-5.5444351257591942 \times 10^{-8}$ \\
34 & $-1.9217495103716011 \times 10^{-7}$ & $-1.9217332626512755 \times 10^{-7}$ \\
35 & $-2.8879775065048592 \times 10^{-7}$ & $-2.8879064153501508 \times 10^{-7}$ \\
44 & $1.3028359742552897 \times 10^{-7}$ & $1.3028391615667287 \times 10^{-7}$ \\
45 & $2.1695644783532599 \times 10^{-8}$ & $2.1693234709859794 \times 10^{-8}$ \\
55 & $-1.8092540754710937 \times 10^{-7}$ & $-1.8092827923235988 \times 10^{-7}$ \\
\hline
\end{tabular}

Table 4: Second derivative of Green's function $U_{J K, 12}$ at evaluation point $\mathbf{x}=(1,1,1)^{T}$ for MEE Material C.

$K$ order rule; and

$$
\mathrm{e}_{S}\left(U_{I J}\right):=\frac{\left\|U_{I J}-U_{I J}^{R}\right\|_{S}}{\left\|U_{I J}^{R}\right\|_{S}},
$$

where $\|\cdot\|_{S}=\int_{S^{2}}|\cdot| \mathrm{d} \omega, S^{2}$ denotes a unit sphere in $\mathbb{R}^{3}$.

The error $e_{\mathrm{int}}^{(K, \alpha)}$ defined in equation (40) is used in order to measure the accumulated error in the integration of the Fourier coefficients for a given number of terms $\alpha$. Figures 2-7 show the evolution of the mean error of all $N \times N$ components $(N=3,4$ or 5 for elastic, PE or MEE) of the Fourier coefficients as

$$
\sum e_{\mathrm{int}}^{(K, \alpha)}:=\frac{\sum_{I=1}^{N} \sum_{J=1}^{N} e_{\mathrm{int}}^{(K, \alpha)}\left(\lambda_{I J}\right)}{N^{2}} .
$$

The reference solution is computed with 20 terms and 256 Gauss points are used in order to obtain Fourier coefficients (21). Figure 2 refers to PE Material A with $\rho=1$. In figure 2 (a) we show in log scale the mean error vs. number of Gauss points used to compute each of the Fourier coefficients (21). Each curve corresponds to a $\alpha$ number of terms included. The same data are shown in figure 2 (b), this time as a family of curves of constant number of $K$ Gauss points as a function of the number of terms $\alpha$. It can be observed that if 64 Gauss points are used the error remains below $10^{-10}$ for any number of terms included in the series. Figure 3 shows the same evolution of the error for the same material but using the formulation with 


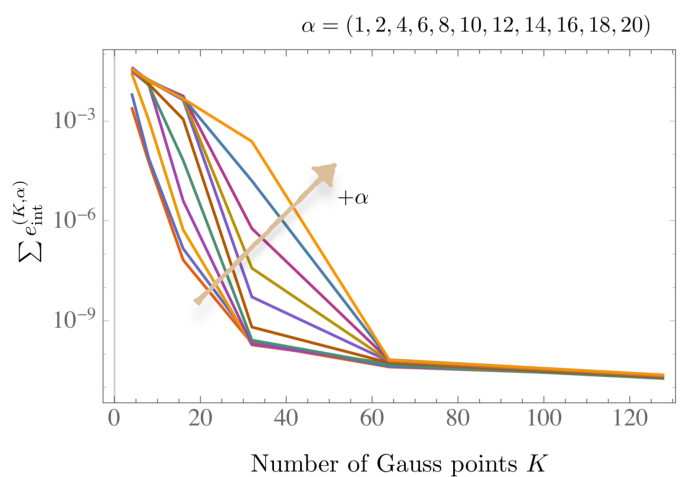

(a)

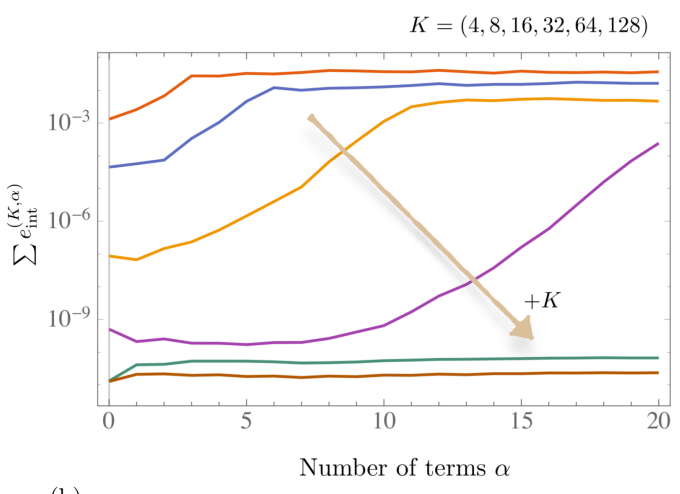

(b)

Figure 2: Mean $e_{\text {int }}^{(K, \alpha)}$ for 16 components the fundamental solution for Material A. Formulation for $\rho=1$. (a) Error vs. number of Gauss points. (b) Error vs. number of terms in the series

$\rho=2$. Comparison with previous figure 2 lead to the conclusion that less Gauss points are needed for the same accuracy if the present formulation with $\rho=2$ for the Fourier series approach is used. Figures 4-7 presents similar results for materials B and C. These numerical tests suggest that -for practical applications- 64 Gauss points are sufficient to evaluate accurately the Fourier coefficients (21), in agreement with [29].

In order to show the convergence behaviour of the Fourier series approach for $\rho=1$ and $\rho=2$, figures 8-10 illustrate in log scale the mean values of the error $\sum \mathrm{e}_{S}$ for Materials $\mathrm{A}, \mathrm{B}$ and $\mathrm{C}$, respectively. In this case the error is defined as

$$
\sum \mathrm{e}_{S}:=\frac{\sum_{J=1}^{N} \sum_{I=1}^{J} \mathrm{e}_{S}\left(U_{I J}\right)}{\frac{1}{2} N(N+1)} .
$$

For all cases, Fourier coefficients (21) have been computed with 128 Gauss points. As a reference for computing $\mathrm{e}_{S}$ the non-degenerate solution given by (7), (18) and (19) has been used. The integration on the unit sphere $S^{2}$ has been performed with standard double Gaussian quadrature with $64 \times 64$ points. The three figures illustrate the fast convergence that shows the Fourier expansion by taking $\rho=2$ when compared with convergence for $\rho=1$. It is shown that higher accuracy is obtained for a given cut-off of the series, or equivalently, for a given degree of accuracy less terms need to be included into the series by taking into account this simple detail on the periodicity of the Barnett-Lothe tensor. 


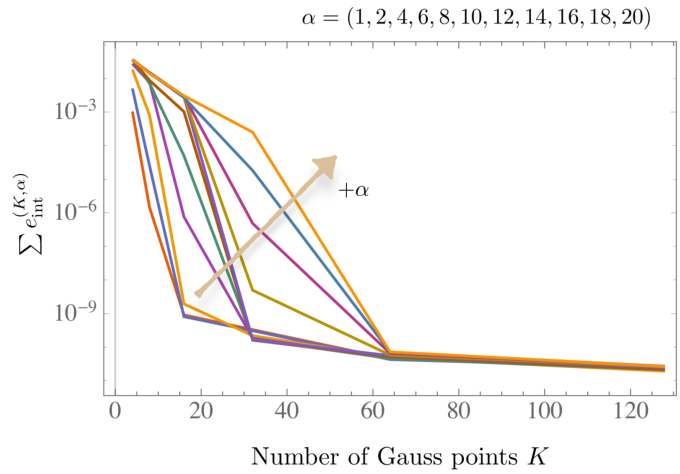

(a)

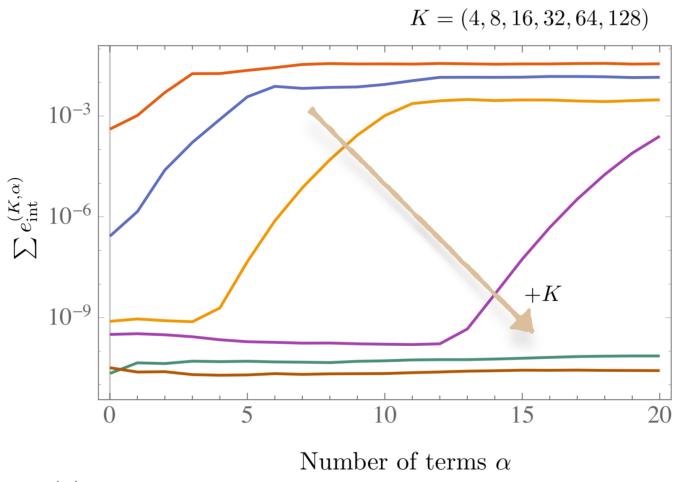

(b)

Figure 3: Mean $e_{\text {int }}^{(K, \alpha)}$ for 16 components the fundamental solution for Material A. Formulation for $\rho=2$. (a) Error vs. number of Gauss points. (b) Error vs. number of terms in the series

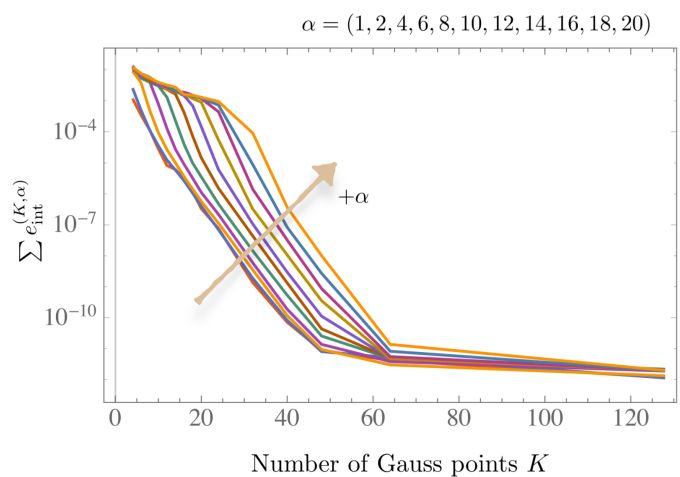

(a)

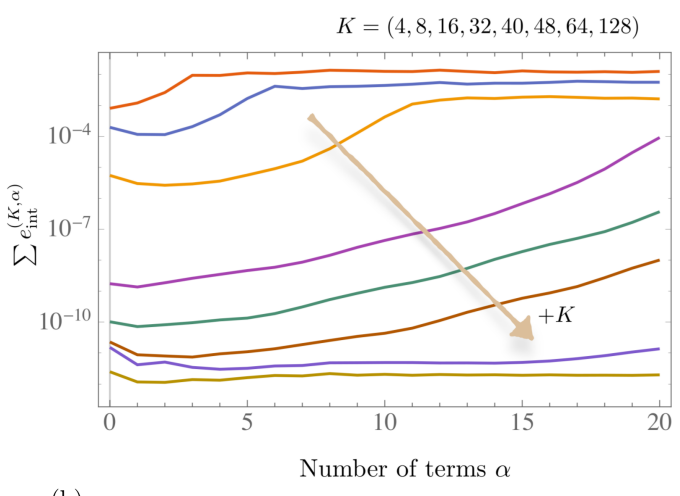

(b)

Figure 4: Mean $e_{\text {int }}^{(K, \alpha)}$ for 25 components the fundamental solution for Material B. Formulation for $\rho=1$. (a) Error vs. number of Gauss points. (b) Error vs. number of terms in the series 


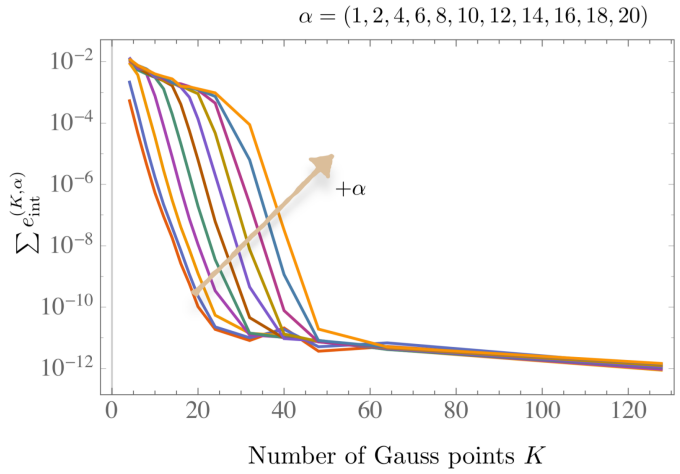

(a)

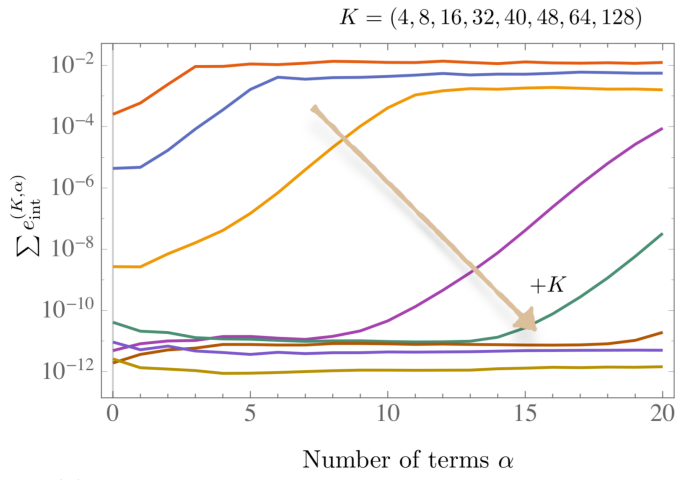

(b)

Figure 5: Mean $e_{\text {int }}^{(K, \alpha)}$ for 25 components the fundamental solution for Material B. Formulation for $\rho=2$. (a) Error vs. number of Gauss points. (b) Error vs. number of terms in the series

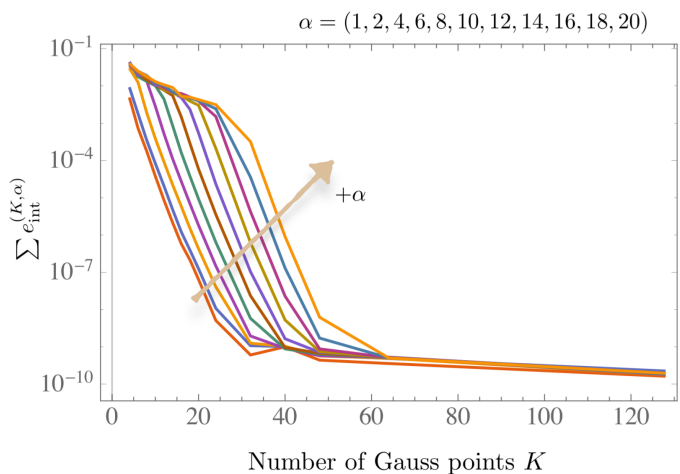

(a)

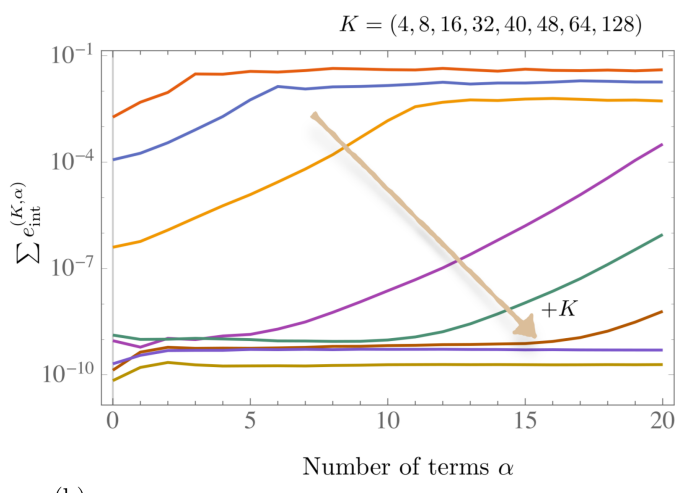

(b)

Figure 6: Mean $e_{\text {int }}^{(K, \alpha)}$ for 25 components the fundamental solution for Material C. Formulation for $\rho=1$. (a) Error vs. number of Gauss points. (b) Error vs. number of terms in the series 


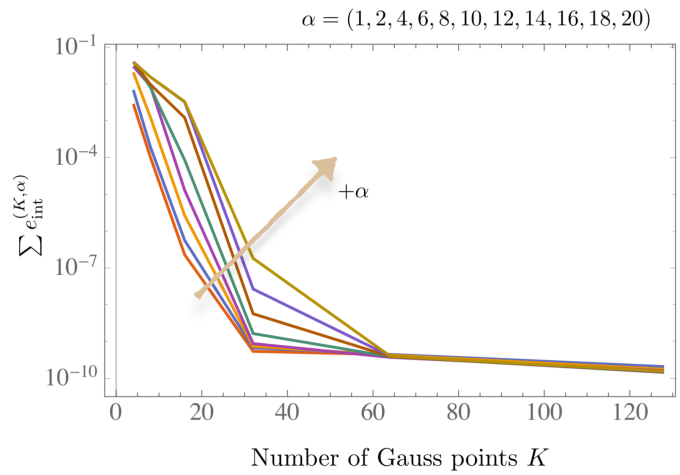

(a)

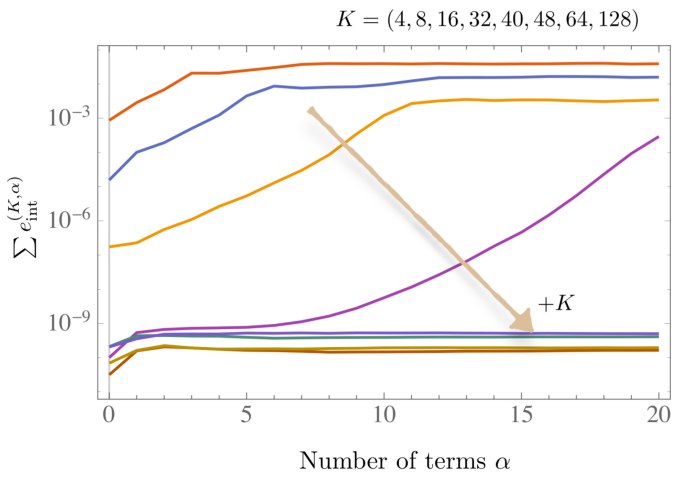

(b)

Figure 7: Mean $e_{\text {int }}^{(K, \alpha)}$ for 25 components the fundamental solution for Material C. Formulation for $\rho=2$. (a) Error vs. number of Gauss points. (b) Error vs. number of terms in the series

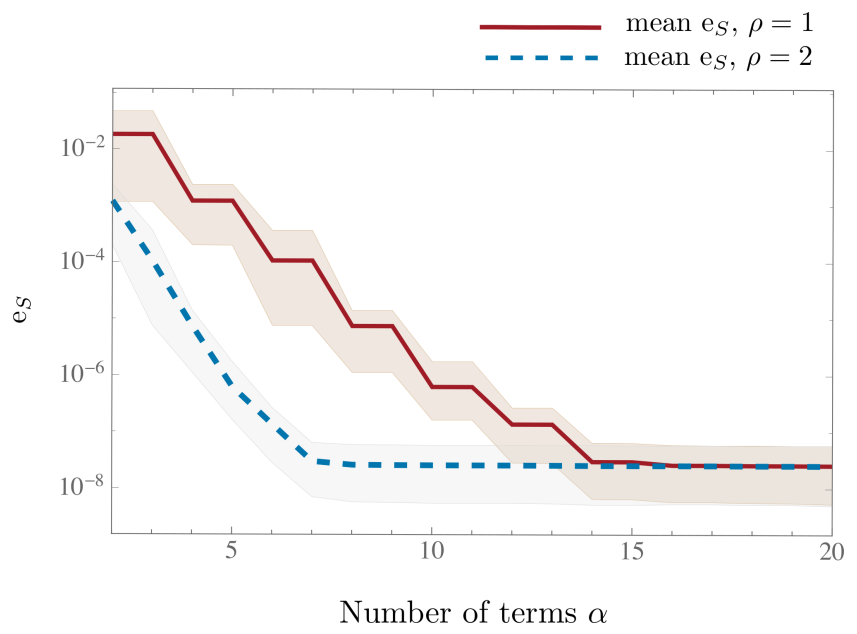

Figure 8: Mean values $\sum \mathrm{e}_{S}$ of the 10 different components the fundamental solution for Material A. Formulation for $\rho=1,2$. 


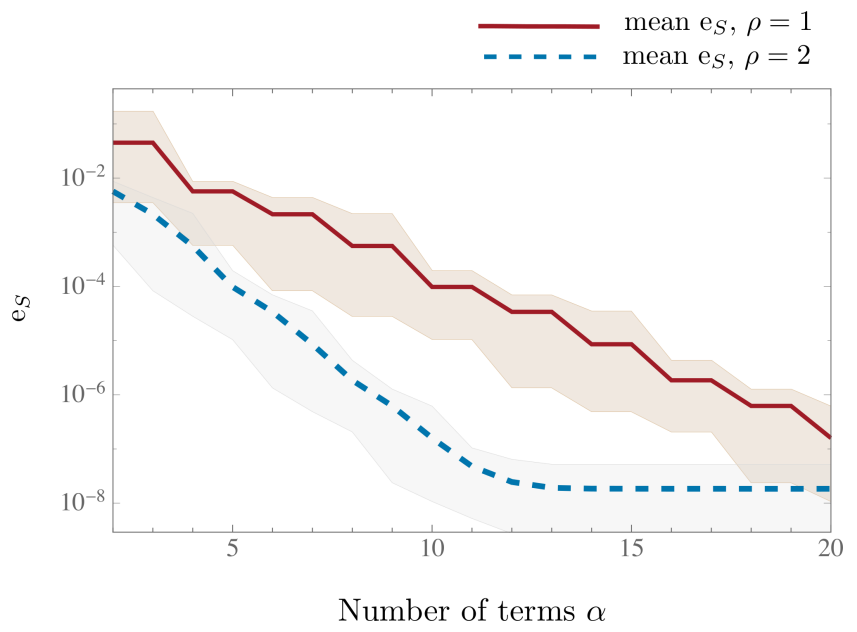

Figure 9: Mean values $\sum \mathrm{e}_{S}$ of the 15 different components of the fundamental solution for Material B. Formulation for $\rho=1,2$.

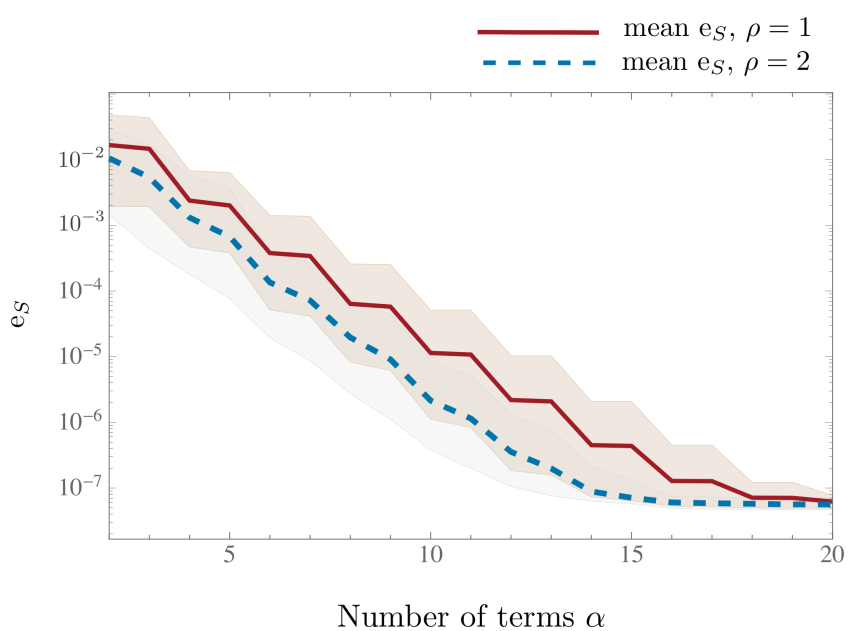

Figure 10: Mean values $\sum \mathrm{e}_{S}$ of the 15 different components of the fundamental solution for Material C. Formulation for $\rho=1,2$. 


\section{Conclusions}

The 3D extended displacement fundamental solution and its first- and second-order derivatives for PE, PM and MEE materials have been obtained and its effective implementation further discussed in this paper. The new expression for the fundamental solution is $(i)$ explicit in terms of the Stroh's eigenvalues, (ii) it remains well-defined when some Stroh's eigenvalues are equal (mathematical degeneracy) or nearly equal (quasi-mathematical degeneracy), and (iii) it is exact. We realise that the mathematical degeneracy presented in previous explicit formulations can be removed by factorization of the denominator in eq. (16), since this comes from the mathematical structure of the solution and not from physical arguments. This solution is used as building block for the development of an alternative efficient approach based on double Fourier series representations. The Fourier series representation is possible due to the periodic nature of the solution. The main benefit from this series solution is that the information of the material properties is contained only in the Fourier coefficients, while the information of the dependence of the evaluation point position is contained in simple trigonometric functions. This results in two advantages: first, any order derivatives can be determined by simple spatial differentiation of the trigonometric functions. We present results for first- and second-order but higher-order derivatives can be built in a straightforward way using this methodology if required. Second, the Fourier coefficients need to be obtained only once for a given material, leading to a very efficient methodology for numerical implementations. We have shown that, exploiting the $\pi$-periodicity of variable $\phi$, better accuracy is obtained for a given number of terms, i.e. convergence is improved (in some cases by several orders of magnitude). Fourier expansion representation is real-valued, which is an important feature for numerical applications. The robustness of the scheme arise from the fact that, due to the properties $(i)$ and $(i i)$ of the new expression for the displacement fundamental solution, the Fourier coefficients can be computed for any general anisotropic coupled material with any kind of mathematical degeneracy in the Stroh context.

In summary, fundamental solutions for anisotropic materials deal with two main drawbacks, the mathematical degeneracy and its overly complex and computationally expensive structure. In this work we developed a scheme for the evaluation of 3D fully anisotropic fundamental solution for MEE materials merging the best of two worlds: the clean structure of the Stroh formalism along with the simplicity of Fourier expansions. These developments 
are expected to help to mitigate the mentioned old drawback of fundamental solutions.

\section{Acknowledgements}

The authors would like to dedicate this work to the memory of Prof. Carlos A. Brebbia (1938-2018), whose relevant research contributions helped to forge the Boundary Element Method and were key in its further development.

This work was supported by the Ministerio de Economía, Industria y Competividad of Spain under project DPI2017-89162-R. R.J.M. would like to acknowledge $\mathrm{CNPq}$, project no.310649, for the support.

\section{Appendix A. Computation of the Stroh's eigenvalues}

In this work, the Stroh's eigenvalues are obtained numerically by solving the linear eigen-problem [26]:

$$
\left(\begin{array}{ll}
\mathbf{N}_{1} & \mathbf{N}_{2} \\
\mathbf{N}_{3} & \mathbf{N}_{1}^{T}
\end{array}\right)\left(\begin{array}{l}
\mathbf{a} \\
\mathbf{b}
\end{array}\right)=p\left(\begin{array}{l}
\mathbf{a} \\
\mathbf{b}
\end{array}\right)
$$

where

$$
\mathbf{N}_{1}=-\mathbf{T}^{-1} \mathbf{R}^{T}, \quad \mathbf{N}_{2}=\mathbf{T}^{-1}, \quad \mathbf{N}_{3}=\mathbf{R} \mathbf{T}^{-1} \mathbf{R}^{T}-\mathbf{Q},
$$

with $\mathbf{Q}, \mathbf{R}$ and $\mathbf{T}$ defined in (13) and the superscript $T$ denoting transpose. In this implementation the GEEVX subroutine of LAPACK library has been used in order to compute the corresponding eigenvalues. Then, the five complex eigenvalues with positive imaginary part are the so-called Stroh's eigenvalues. The remainder fives are their complex conjugates.

\section{Appendix B. Materials}

In this appendix we summarize the material properties used in this work. Materials A and B are transversely isotropic and therefore satisfied the following relations:

$$
\begin{gathered}
c_{1212}=\frac{c_{1111}-c_{1122}}{2}, \quad c_{1313}=c_{2323}, \quad c_{2222}=c_{1111}, \quad c_{2233}=c_{1133} \\
e_{322}=e_{311}, \quad e_{223}=e_{113}, \quad q_{322}=q_{311}, \quad q_{223}=q_{113} \\
\lambda_{22}=\lambda_{11}, \quad \epsilon_{22}=\epsilon_{11}, \quad \mu_{22}=\mu_{11} .
\end{gathered}
$$

Non-vanishing components for Materials A, B and C are presented in Tables B.5, B.6 and B.7, respectively. 
Table B.5: Piezoelectric properties for Material A

\begin{tabular}{ccc}
\hline & $c_{1111}$ & 139 \\
Elastic constants $\left[10^{9} \frac{\mathrm{N}}{\mathrm{m}^{2}}\right]$ & $c_{3333}$ & 115 \\
& $c_{1122}$ & 77.8 \\
& $c_{1133}$ & 74.3 \\
Piezoelectric constants $\left[\frac{\mathrm{C}}{\mathrm{m}^{2}}\right]$ & $c_{2323}$ & 25.6 \\
\hline \multirow{2}{*}{ Dielectric permeability coefficients $\left[10^{-9} \frac{\mathrm{C}}{\mathrm{Vm}}\right]$} & $e_{113}$ & 12.7 \\
& $e_{333}$ & 15.1 \\
& $e_{322}$ & -5.1 \\
\hline
\end{tabular}

Table B.6: Magneto-electro-elastic properties for Material B

\begin{tabular}{|c|c|c|}
\hline \multirow{5}{*}{ Elastic constants $\left[10^{9} \frac{\mathrm{N}}{\mathrm{m}^{2}}\right]$} & $c_{1111}$ & 166 \\
\hline & $c_{3333}$ & 77 \\
\hline & $c_{1122}$ & 78 \\
\hline & $c_{1133}$ & 162 \\
\hline & $c_{2323}$ & 43 \\
\hline \multirow{3}{*}{ Piezoelectric constants $\left[\frac{C}{m^{2}}\right]$} & $e_{113}$ & 11.6 \\
\hline & $e_{333}$ & 18.6 \\
\hline & $e_{322}$ & -4.4 \\
\hline \multirow{3}{*}{ Piezomagnetic constants $\left[\frac{N}{A m}\right]$} & $q_{113}$ & 550 \\
\hline & $q_{333}$ & 699.7 \\
\hline & $q_{322}$ & 580.3 \\
\hline \multirow{2}{*}{ Magneto-electric coefficients $\left[\frac{N s}{A m}\right]$} & $\lambda_{11}$ & 0 \\
\hline & $\lambda_{33}$ & 0 \\
\hline \multirow{2}{*}{ Dielectric permeability coefficients $\left[10^{-9} \frac{C}{V m}\right]$} & $\epsilon_{11}$ & 11.2 \\
\hline & $\epsilon_{33}$ & 12.6 \\
\hline \multirow{2}{*}{ Magnetic permeability coefficients $\left[10^{-6} \frac{\mathrm{Ns}^{2}}{\mathrm{C}^{2}}\right]$} & $\mu_{11}$ & 5 \\
\hline & $\mu_{33}$ & 10 \\
\hline
\end{tabular}


Table B.7: fully magneto-electro-elastic properties for Material C

\begin{tabular}{|c|c|c|c|c|c|c|}
\hline \multirow{7}{*}{ Elastic constants $\left[10^{9} \frac{\mathrm{N}}{\mathrm{m}^{2}}\right]$} & $c_{1111}$ & 220.12 & $c_{1112}$ & 2.09 & $c_{1113}$ & -2.196 \\
\hline & $c_{1122}$ & 124.285 & $c_{1123}$ & -0.853 & $c_{1133}$ & 125.807 \\
\hline & $c_{2222}$ & 218.338 & $c_{1212}$ & 46.651 & $c_{1213}$ & 0.552 \\
\hline & $c_{1222}$ & 0.991 & $c_{1223}$ & -0.968 & $c_{1233}$ & -1.317 \\
\hline & $c_{1333}$ & -0.505 & $c_{1322}$ & 1.115 & $c_{1323}$ & -0.217 \\
\hline & $c_{2223}$ & 1.516 & $c_{2233}$ & 126.24 & $c_{2333}$ & 0.401 \\
\hline & $c_{3333}$ & 215.586 & $c_{2323}$ & 45.753 & $c_{1313}$ & 46.258 \\
\hline \multirow{6}{*}{$\begin{array}{l}\text { Piezoelectric constants } \\
{\left[\frac{\mathrm{C}}{\mathrm{m}^{2}}\right]}\end{array}$} & $e_{111}$ & 9.374 & $e_{112}$ & -4.666 & $e_{113}$ & 8.847 \\
\hline & $e_{122}$ & -1.767 & $e_{123}$ & -0.820 & $e_{133}$ & -0.643 \\
\hline & $e_{211}$ & 1.241 & $e_{212}$ & 4.857 & $e_{213}$ & -0.821 \\
\hline & $e_{222}$ & -5.721 & $e_{223}$ & 7.441 & $e_{233}$ & 0.519 \\
\hline & $e_{311}$ & -2.354 & $e_{312}$ & -0.504 & $e_{313}$ & 5.379 \\
\hline & $e_{322}$ & -1.395 & $e_{323}$ & -4.346 & $e_{333}$ & 13.214 \\
\hline \multirow{6}{*}{$\begin{array}{l}\text { Piezomagnetic constants } \\
{\left[\frac{N}{A m}\right]}\end{array}$} & $q_{111}$ & 358.82 & $q_{112}$ & -61.593 & $q_{113}$ & 116.775 \\
\hline & $q_{122}$ & 130.939 & $q_{123}$ & 26.878 & $q_{133}$ & 94.107 \\
\hline & $q_{211}$ & -99.692 & $q_{212}$ & 44.217 & $q_{213}$ & 26.881 \\
\hline & $q_{222}$ & -200.163 & $q_{223}$ & 67.743 & $q_{233}$ & -76.044 \\
\hline & $q_{311}$ & 189.018 & $q_{312}$ & 11.224 & $q_{313}$ & 37.090 \\
\hline & $q_{322}$ & 135.735 & $q_{323}$ & -29.976 & $q_{333}$ & 291.03 \\
\hline \multirow{2}{*}{$\begin{array}{l}\text { Magneto-electric } \\
\text { coefficients }\left[\frac{N s}{A m}\right]\end{array}$} & $\lambda_{11}$ & -0.688 & $\lambda_{12}$ & 0.413 & $\lambda_{13}$ & -0.784 \\
\hline & $\lambda_{22}$ & -1.022 & $\lambda_{23}$ & 0.634 & $\lambda_{33}$ & -1.890 \\
\hline \multirow{2}{*}{$\begin{array}{l}\text { Dielectric permeability } \\
\text { coefficients }\left[10^{-9} \frac{C}{V m}\right]\end{array}$} & $\epsilon_{11}$ & -5.817 & $\epsilon_{12}$ & 0.107 & $\epsilon_{13}$ & -0.203 \\
\hline & $\epsilon_{22}$ & -5.905 & $\epsilon_{23}$ & 0.165 & $\epsilon_{33}$ & -6.129 \\
\hline \multirow{2}{*}{$\begin{array}{l}\text { Magnetic permeability } \\
\text { coefficients }\left[10^{-6} \frac{\mathrm{Ns}^{2}}{\mathrm{C}^{2}}\right]\end{array}$} & $\mu_{11}$ & -243.622 & $\mu_{12}$ & -32.340 & $\mu_{13}$ & 61.326 \\
\hline & $\mu_{22}$ & -217.516 & $\mu_{23}$ & -49.543 & $\mu_{33}$ & -149.639 \\
\hline
\end{tabular}




\section{References}

[1] J. Ma, J. Hu, Z. Li, C-W Nan (2011) Recent Progress in Multiferroic Magnetoelectric Composites: from Bulk to Thin Films, Advanced Materials. 23, 1062-1087

[2] Y. Wang, J. Li, D. Viehland (2014) Magnetoelectrics for magnetic sensor applications: status, challenges and perspectives, Materials Today, 17 (6)

[3] C.A. Brebbia (1978) The boundary element method for engineers. London/New York: Pentech Press/Halstead Press

[4] C.A. Brebbia, J.C.F. Telles, L.C. Wrobel (1984) Boundary Element Techniques: Theory and Applications in Engineering. Springer-Verlag

[5] C.A. Brebbia, J. Dominguez (1992) Boundary elements: An introductory course. WIT Press

[6] A. Cheng, D.T. Cheng (2005) Heritage and early history of the boundary element method. Engineering Analysis with Boundary Elements 29, 268302

[7] M.M. Muñoz-Reja, F.C. Buroni, A. Sáez, F. García-Sánchez (2016) 3D Explicit-BEM Fracture Analysis for Materials with Anisotropic Multifield Coupling. Applied Mathematical Modelling. 40, 2897-2912

[8] X. Han (2009) High-order derivatives of Green's functions in magnetoelectro-elastic materials. International Journal of Solids and Structures $46,3405-3411$

[9] C.Y. Wang, J. D. Achenbach (1995) Three-dimensional time-harmonic elastodynamic Green's functions for anisotropic solids, Proceedings of the Royal Society A: Mathematical, Physical Sciences, 449, pp. 441-458.

[10] F.C. Buroni, M. Denda (2014) Radon-Stroh formalism for 3D theory of anisotropic elasticity, In Proceedings of the 15th International Conference on Boundary Element \& Meshless Techniques, BETEQ, V. Mallardo, M.H. Aliabadi (eds). Florence 
[11] E. Pan (2002) Three-dimensional Green's functions in anisotropic magneto-electro-elastic bimaterials, Zeitschrift für angewandte Mathematik und Physik ZAMP. 53, 815-838

[12] F.C. Buroni, A. Sáez (2010) Three-dimensional Green's function and its derivative for materials with general anisotropic magneto-electro-elastic coupling. Proceedings of the Royal Society A: Mathematical, Physical Sciences 466 515-537.

[13] L. Xie, Ch. Zhang, C. Hwu, J. Sladek, V. Sladek (2015) On two accurate methods for computing 3D Green's function and its first and second derivatives in piezoelectricity. Engineering Analysis with Boundary Elements, 61, 183-193

[14] L. Xie, Ch. Zhang, C. Hwu, E. Pan (2016) On novel explicit expressions of Green's function and its derivatives for magnetoelectroelastic materials. European Journal of Mechanics A/Solids 60, 134-144

[15] L. Xie, Ch. Zhang, J. Wang (2018) Unified and explicit expressions of three-dimensional Green's functions and their first derivatives for piezoelectric solids with general anisotropy, International Journal of Solids and Structures doi: 10.1016/j.ijsolstr.2018.05.009

[16] C.-L. Hsu, C. Hwu, Y.C. Shiah (2019) Three-dimensional boundary element analysis for anisotropic elastic solids and its extension to piezoelectric and magnetoelectroelastic solids. Engineering Analysis with Boundary Elements 98, 265-280

[17] K-C. Wu (1998) Generalization of the Stroh Formalism to 3-Dimensional Anisotropic Elasticity. Journal of Elasticity 51, 213-225

[18] R. Wilson, T. Cruse (1978) Efficient implementation of anisotropic three dimensional boundary-integral equation stress analysis. International Journal of Numerical Methods in Engineering, 12, 1383-1397

[19] S. Muraishi (2016) Efficient interpolation algorithm of electro-elastic Green's function for boundary integral equation method and Eshelby inclusion problem. International Journal of Solids and Structures. 100101, 297-306 
[20] V. Gulizzi, A. Milazzo, I. Benedetti (2016) Fundamental solutions for general anisotropic multi-field materials based on spherical harmonics expansions. International Journal of Solids and Structures. 100-101, 169186

[21] V.I. Fabrikant (2017) Green's functions for the magneto-electro-elastic anisotropic half-space and their applications to contact and crack problems. Archive of Applied Mechanics. 87, 1859-1869

[22] I. Pasternak, V. Pasternak, R. Pasternak, H. Sulym (2017). Stroh formalism in evaluation of 3D Green's function in thermomagnetoelectroelastic anisotropic medium. Mechanics Research Communications. 84, 2026

[23] D.M. Barnett, J. Lothe (1975) Dislocations and Line Charges in Anisotropic Piezoelectric Insulators. Physica Status Solidi (b) 67, 105111

[24] V.I. Alshits, A.N. Darinskii, J. Lothe(1992) On the existence of surface waves in half-infinite anisotropic elastic media with piezoelectric and piezomagnetic properties. Wave Motion 16 265-283

[25] Fan, H. Special Topics in the Theory of Piezoelectricity, Chapter 3: Twodimensional static problems: Stroh formalism. Ed. Yang, J. Springer New York 2009

[26] T.C.T. Ting. 1996 Anisotropic Elasticity, Oxford University Press, Oxford.

[27] T.C.T. Ting, V.G. Lee (1997) The three-dimensional elastostatic Green's function for general anisotropic linear Elastic solids, The Quarterly Journal of Mechanics and Applied Mathematics. 50, 407-426

[28] Wolfram Research, Inc., Mathematica, Version 11.3, Champaign, IL (2018).

[29] Y.C. Shiah, C.L. Tan, C.Y. Wang (2012) Efficient computation of the Green's function and its derivatives for three-dimensional anisotropic elasticity in BEM analysis. Engineering Analysis with Boundary Elements $36,1746-1755$ 
[30] C.L. Tan, Y.C. Shiah, C.Y. Wang (2013) Boundary element elastic stress analysis of 3D generally anisotropic solids using fundamental solutions based on Fourier series. International Journal of Solids and Structures 50, 2701-2711

[31] C.L. Tan (2018) Private communication. 